\title{
Los usuarios de las bibliotecas de la Universidad Nacional Mayor de San Marcos
}

\author{
Julio César Olaya Guerrero \\ Departamento Académico de Bibliotecología \\ y Ciencias de la Información
}

\section{Introducción}

T a literatura especializada define al usuario como: "aquél individuo

L que necesita información para desarrollar las actividades". ${ }^{1}$ Este concepto está relacionado con la demanda de información, que se define como "la formulación expresa de un deseo, o dicho de otro modo, es lo que un individuo solicita a un Centro de Documentación y/o Biblioteca. ${ }^{2}$

Otro concepto importante es la tipología de usuarios. Elías Sanz (1994) nos presenta dos tipos de usuarios:

a) Usuarios Potenciales: Son aquellos que necesitan información, pero no son conscientes de ello:

b) Usuarios Reales: Son aquellos que no solo son conscientes que necesitan información sino que fautilizan frecuentemente.

En las Bibliotecas Universitarias aparecen estos dos tipos de usuarios; además, existen la categoría de docentes, alumnos, personal administrativo, otros (incluye lectores visitantes de otras universidades, investigadores).

Otro concepto importante son las necesidades de información. Según Juan José Calva (1996), las necesidades de información son un fenómeno que continuamente tiene el ser humano contemporáneo en el desarrollo de todas sus actividades, que como ente social realiza, ya sean éstas: educativas, laborales, políticas, económicas, etc.

\footnotetext{
1 Elías Sanz. Manual de estudios de usuarios. Madrid: Fundación Germám Sénchez, 1994. p. 19.

2 Elias Sanz. Op. cit., p. 25.
} 
Para lograr satisfacer las necesidades de información de una persona o un grupo de personas integrantes de una comunidad se deben identificar tales necesidades y diseñar y establecer los mecanismos adecuados para satisfacerlas.

Hoy en día las bibliotecas de investigación ya están compartiendo algunos elementos relacionados con sus objetivos, con otros colaboradores importantes en el campus: los centros de cómputo y de telecomunicaciones que tienen que mantener el sistema circulatorio de la universidad y que son los proveedores clave de la infraestructura y el soporte tecnológico. ${ }^{3}$

Es importante remarcar que "las bibliotecas académicas enfrentan y continuarán enfrentando una espiral de cambios que significarán enormes desafíos para la planeación y la puesta en práctica de soluciones radicales". ${ }^{4}$

Muchas veces, acceder a la información sugiere un costo. Entonces ¿Pagar por el acceso constituye una barrera? Espontáneamente, me gustaría contestar no. De hecho si necesitola información lo justo es que pague por ella. Esta es la respuesta de un investigador que tiene un subsidio y recursos para pagar, pero ¿qué pasa con los estudiantes, en particular con los de licenciatura, que es otra categoría de usuarios frecuentemente olvidada en nuestro desarrollo estratégico de nuevos servicios? ¿Puede o debe pagar? El costo constituye definitivamente un factor limitante, a menos de que negociemos convenios con los proveedores de informacióntebien subsídiemosel acceso de los estudiantes. Actualmente, la Universidad de SaniMarcos cuentason el Sistema de Bibliotecas y catálogo en línea, que permite el acceso a la información desde cualquier lugar del Perú.

\section{Análisis de los datos}

\subsection{Consultas por tipo de usuario en las bibliotecas de la UNMSM}

A fines de la década de los ochenta, la Universidad de San Marcos solicitaba en forma constante al gobierno de turno, mayor equipamiento de laboratorios, bibliotecas y rentas para poder cumplir su misión. Las bibliotecas de la Universidad siguieron brindando los servicios en forma normal, salvo algunas

\footnotetext{
"Khouri, Anastassia. "Reingeniería de los servicios en las bibliotecas universitarias". En: Investigación Bibliotecológica, vol. 11, N²2, Ene-Jun, 1997, p. 64.

4 Khouri, Anastassia. Op. cit., p. 65.
} 
paralizaciones.

En 1988, el total de usuarios de la bibliotecas de la Universidad fue de 869 541; la categoría alumnos reportó 778 180. Mientras que en 1989, el total fue de 1093131 personas, destacando los usuarios alumnos con 942 630, aumentando en 164450 personas en relación a 1988.

\section{Cuadro $N^{\circ} 1$}

CONSULTAS POR TIPO DE USUARIO EN LAS BIBLIOTECAS DE UNMSM

$1988-2000$

\begin{tabular}{|c|c|c|c|c|c|}
\hline Años & Alumnos & Docentes & Administra & Otros & Total \\
\hline 1988 & 778180 & 33891 & 6676 & 50794 & 869541 \\
\hline 1989 & 942630 & 43903 & 12316 & 94282 & 1093131 \\
\hline 1990 & 765602 & 37974 & 6472 & 93887 & 903935 \\
\hline 1991 & 645579 & 33282 & 7014 & 75513 & 761388 \\
\hline 1992 & 864060 & 41358 & 7335 & 68144 & 980897 \\
\hline 1993 & 712722 & 28936 & 5628 & 61008 & 808294 \\
\hline 1994 & 564132 & 27430 & 6470 & 42808 & 641020 \\
\hline 1995 & 1186165 & 54171 & 8948 & 102721 & 1352005 \\
\hline 1996 & 1027392 & 50888 & 7707 & 119032 & 1205019 \\
\hline 1997 & 1355019 & 33927 & 5360 & 229028 & 1623334 \\
\hline 1998 & 1377965 & 28032 & 4735 & 229861 & 1640593 \\
\hline 1999 & 1379734 & 22487 & 2942 & 192365 & 1597528 \\
\hline 2000 & 1289618 & 23277 & 3791 & 208599 & 1525285 \\
\hline
\end{tabular}

Fuente: Boletin Estadístico Nos. 7, 8 y 9, UNMSM-OGP. 1993, 1994 y 1997. Pp. 81-83, 97 y 113 respectivamente.

Compendio Estadístico 1996, 1997, 1998, 1999. UNMSM-OGP, Nos. 10, 11, 12 y 13. Pp. 131, 125. 183, 209 respectivamente. Compendio Estadístico 2000. UNMSM-OGP. Vers. Electrón.

Elaboración del autor. 
En 1990, el total de personas que accedieron a las bibliotecas fue de 903935 usuarios; la categoría de alumnos arrojó 765602 , mientras que los docentes registró 37 974. A continuación, se presenta el Cuadro $\mathrm{N}^{\circ} 1$, que registra el tipo de usuarios de la Universidad y sus valores correspondientes, entre 1988 y 2000.

En la década de los noventa, luego de una ligera baja en la categoría usuarios alumnos de 564132 en 1994; se produce un incremento significativo de 622033 personas, llegando a 1186165 para 1995. Estos datos varían ligeramente entre 1997 y 1999. En el año 2000, el número de usuarios alumnos fue de 1289618 . Mientras que la categoría docentes registró 27430 en 1994; en 1995, se incrementó a 54171 personas. Para 1997, bajo el acceso de los docentes con solamente 22487 . La categoria otros (donde suelen aparecer los lectores visitantes de otras universidades, investigadores), presenta su nivel más alto en 1998, con 229861 personas. Disminuyendo en el año 2000 a 208599 lectores visitantes.

2.2. Consulta global por tipo de naterial bibliográfico en las bibliotecas de UNMSM

Hemos obtenido para los años 1970 y 1980 , valores anuales de la consulta global de material bibliográfico en las bibliotecas de la Universidad de San Marcos. En el período señala doterc1474se consultaron 2000560 entre libros, revistas, tesis. En 1978 losvalores sémeodificantotabizando solamente 1876362 consultas; en 1979, los valores totales siguieron disminuyendo hasta llegar a 1602758 consultas. A continuación se presenta el Cuadro $\mathrm{N}^{\circ} 2$.

En la década de los ochenta, se evidencia que el material consultado aumenta en forma significativa. Para 1982, significó 1843732 consultas, entre libros, folletos tesis y revistas; en 1983, el material bibliográfico fue de 2116943 libros, 22671 folletos, 94959 tesis y 96472 revistas, totalizaron 2331050 consultas. Luego, en 1984, el total de consultas en las diferentes bibliotecas de la Universidad fuc de: 808 323. Para los años 1985 y 1987 no se posee información al respecto.

En 1988, el número de consultas fue de 1058 872; es importante señalar que tuvieron mayor acogida entre los usuarios: libros y revistas. Para 1989, el total de consultas aumentó a 1308855 , esto significa 249983 solicitudes más que el año anterior. Terminando la década, el total de consultas para 1990 fue de 912 575; disminuyendo 396280 solicitudes en relación a 1989. Esto debido a 


\section{CUADRO $N^{\circ} 2$ \\ CONSULTA GLOBALDE MATERIAL BIBLIOGRAFICO \\ BIBLIOTECAS DE LA UNMSM \\ 1970-1980}

\begin{tabular}{|c|c|}
\hline AÑO & $\begin{array}{c}\text { Material bibliográfico } \\
\text { (Valores anuales) }\end{array}$ \\
\hline 1970 & 893968 \\
\hline 1971 & 1145927 \\
\hline 1972 & 1253757 \\
\hline 1973 & 1678676 \\
\hline 1974 & 2000560 \\
\hline 1975 & 1750578 \\
\hline 1976 & 1890702 \\
\hline 1977 & 1802112 \\
\hline 1978 & 1876362 \\
\hline 1979 & 1602758 \\
\hline 1980 & 1649417 \\
\hline
\end{tabular}

Fuente: Memoria 1980. UNMSM. Lima: 1981. p. 129.

Elaboración: del autor.

problemas laborales (huelga de docentes y trabajadores). A continuación se presenta el Cuadro $\mathrm{N}^{\circ} 3$.

\section{"Jorge Puccinelli Converso»}

En el período 1991 y 1999, el número de consultas evidenció grandes diferencias, debido a falta de personal, bibliotecas cerradas por inventario, se fusiona la Bibliioteca Central y la hemeroteca en 1997. Para una mejor comprensión, vayamos al Cuadro $\mathrm{N}^{\circ} 4$.

En la década de los Noventa, el mayor número de consultas se registró en los años 1992 con 1028 514, en 1995 fue de 1760482 y 2194683 , en 1999. Mostrando una gran disminución en los años 1993 y 1994. Uno de los aspectos importantes que se debe afianzar en las Bibliotecas de la Universidad es la educación de usuarios. 


\section{CUADRO $\mathrm{N}^{\circ} 3$ \\ CONSULTA GLOBALDE MATERIAL BIBLIOGRAFICO \\ BIBLIOTECAS DE LA UNMSM \\ $1982-1990$}

\begin{tabular}{|c|c|}
\hline AÑO & $\begin{array}{c}\text { Material bibliográfico } \\
\text { (Valores anuales) }\end{array}$ \\
\hline 1982 & 1843732 \\
\hline 1983 & 2331050 \\
\hline 1984 & 808323 \\
\hline 1985 & s.d \\
\hline 1986 & s.d \\
\hline 1987 & s.d \\
\hline 1988 & 1058872 \\
\hline 1989 & 1308855 \\
\hline 1990 & 912575 \\
\hline
\end{tabular}

Fuente: Memoria 1982, 1983, 1984/

Gastón Pons Muzzo. UNMSM.

Boletin Estadistico / UNMSM

Lima: OGP, 1992.

\section{Educación de Usuarios en UNMSM Ietras «suarios en UNMSM}

La educación de usuarios en las Bibliotecas de la Universidad es un elemento fundamental, ya que permitirá que los docentes, alumnos y lectores visitantes puedan recibir capacitación sobre el uso de la Biblioteca y sus servicios. Esta capacitación debe ser permanente.

Las bibliotecas deben recibir sugerencias o comentarios para poder mejorar sus servicios; esto puede ser por teléfono, correo electrónico o en forma personal. Asimismo, el personal que brinda el servicio de lectura, internet y préstamo a domicilio necesita un entrenamiento especial.

\section{Biblioteca Digital}

Según Saffady, "una biblioteca digital es una biblioteca que mantiene toda o parte de su colección en forma computarizada, ya sea sustituyendo, com- 


\section{CUADRO N ${ }^{\circ} 4$ \\ CONSULTA GLOBAL DE MATERIAL BIBLIOGRAFICO \\ BIBLIOTECAS DE LA UNMSM \\ $1991-1999$}

\begin{tabular}{|c|c|}
\hline AÑO & $\begin{array}{c}\text { Material bibliográfico } \\
\text { (Valores anuales) }\end{array}$ \\
\hline 1991 & 791805 \\
\hline 1992 & 1028514 \\
\hline 1993 & 870648 \\
\hline 1994 & 647044 \\
\hline 1995 & 1760482 \\
\hline 1996 & s.d \\
\hline 1997 & 1034159 \\
\hline 1998 & 2194683 \\
\hline 1999 & 1638854 \\
\hline
\end{tabular}

Fuente: Boletín Estadístico $\mathrm{N}^{\circ} \%$, UNMSM-OGP. 1993

Boletín Estadistico No 8, UNMSM-OGP. 1994

Boletín Estadistico No 9, UNMSM-OPP. 1995.

Compendio Estadístico 1996. UNMSM-OGP. N 10.

Compendio Estadístico 1997. UNMSM-OGP. $N^{\circ} 11$.

Compendio Estadístico 1998. UNMSM-OGP. $\mathrm{N}^{\circ} 12$.

Compendio Estadistico 1999. UNMSM-OGP. N¹3.

plementando o como alternativa de los materiales tradicionales impresos o microfilmados que usualmente posee la biblioteca.

Algunos autores definen los metadatos como "datos asociados con objetos que ahorran a los usuarios el trabajo de conocer los originales en su totalidad". En un espacio informativo tan vasto como internet, la efectividad de los accesos depende del manejo efectivo que se haga de la información disponible en las redes electrónicas.

\section{Conclusiones}

En la Universidad de San Marcos, los usuarios son principalmente docentes y alumnos; en los últimos años, la Biblioteca Central se ha modernizado y se

5 William Saffady. "Digital library concepts and technologies for the management of library collections: an analysis of methods and costs". Library Technology Reports 31 (May-Jun 1995). 
accede a buscar información bibliográfica, mediante catálogo en línea. En el período estudiado, se evidencia un alto número de consultas de bibliotecas ligadas a Ciencias Médicas y Derecho.

En las actuales circunstancias, las bibliotecas deben ser competitivas, ofreciendo nuevos servicios como DSI (Diseminación Selectiva de Información) para los docentes investigadores y visitantes.

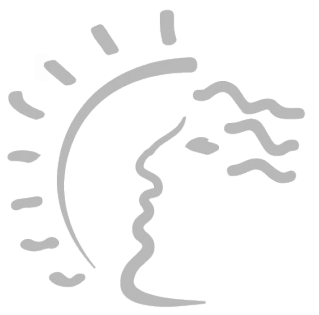

Biblioteca de Letras "Jorge Puccinelli Converso» 